\title{
Eicosanoids in exhaled air after non-specific challenge in patients with asthma
}

\author{
Marek Kaszuba ${ }^{1 *}$, Jagoda Kaszuba' ${ }^{1}$ Agata Sawina' ${ }^{1}$ Agata Kleciek' ${ }^{1}$ Paweł Stręk², Marek Sanak' and Lucyna Mastalerz' \\ *Correspondence: markas@op.pl \\ 'Second Department of Internal Medicine, Jagiellonian University Medical College, Poland. \\ ${ }^{2}$ Department of Otolaryngology, Jagiellonian University Medical College, Poland.
}

\begin{abstract}
Background: Histamine is used as a direct stimulus to measure airway responsiveness. This short-acting biogenic amine acts mainly on airway smooth muscle receptors causing bronchoconstriction and is used for the airway hyperresponsiveness assessment in asthmatic patients. In aspirin-induced asthma (AIA) special regulatory role of eicosanoids is postulated. The aim of the study was to assess the influence of histamine on a wide profile of eicosanoids measured in exhaled breath condensate (EBC) in AIA patients and asthmatics tolerating aspirin well (ATA).

Methods: The study population consisted of seventeen asthmatics. Ten of them were AIA patients. Eicosanoid concentrations in $\mathrm{EBC}$ were determined using gas chromatography/mass spectrometry or high-performance liquid chromatography/tandem mass spectrometry. Measurements were performed at baseline and following bronchial histamine challenge.

Results: Bronchial reactions were precipitated by histamine in all patients and accompanied only by decrease of leukotriene (LT) $\mathrm{C}_{4}$ and trans-LTC 4 mean level. The AIA group was characterized by higher levels of cysteinyl leukotrienes, LTC $_{4}$, and prostaglandin $(\mathrm{PG}) \mathrm{E}_{2}$ in $\mathrm{EBC}$ at baseline, and decrease in $\mathrm{EBC}$ concentration of $\mathrm{LTC}_{4}$, trans-LTC $\mathrm{L}_{4}$ and tetranor-PGE-M following histamine challenge. In the ATA group no significant changes in eicosanoids levels after histamine were noticed. Conclusions: AIA patients present different baseline profile of EBC eicosanoids in comparison to patients with ATA. Histamine administered locally during a bronchial challenge test may influence inflammatory mediators and thus trigger indirect effects in the respiratory tract. This response for histamine differentiates two studied phenotypes of asthma; only in the AIA group histamine precipitates alterations of the eicosanoid synthesis in the lungs.
\end{abstract}

Keywords: Aspirin, asthma, hypersensitivity, eicosanoids, histamine

\section{Introduction}

Histamine is a short-acting biogenic amine which plays an important role in allergic inflammation. Mast cells and basophiles store large quantities of histamine, which is released during degranulation in response to immunologic and nonimmunologic stimuli [1]. Histamine elicits many biological effects acting through four types of histamine receptors located on cells of various types. These include airway smooth muscle cells provoking airflow obstruction and sensory fibers causing a reflex response that can induce bronchospasm. Histamine, beside methacholine, is used as a direct stimulus to measure airway responsiveness.

Airway hyperresponsiveness (AHR) is one of the clinical features of asthma. Its measurement is crucial for establishing the correct diagnosis [17]. The components of airway changes in asthma that contribute to AHR can be differentiated into two categories: airway remodeling and airway inflammation.

It has been suggested that the action of histamine particularly reflects the effect associated with contractility of airway smooth muscle, called a direct effect. To a lesser degree, histamine may affect airway inflammation, which is called an indirect effect. However, the role of histamine in direct and indirect effects has not been clearly established. There is a correlation between measurements of airway responsiveness and number of eosinophils, mast cells [13] and epithelial cells [4] in bronchoalveolar lavage fluid (BALF) in asthma. In our study, we focused on the indirect action of histamine by measuring mediators released from inflammatory cells into the lumen of the airways in response to this agent.

Eicosanoids, including prostaglandins and leukotrienes, are arachidonic acid metabolites which play an important role in chronic inflammation in asthma, especially in the pathogenesis of aspirin intolerant asthma (AIA). AIA is a distinct clinical syndrome which affects $5-10 \%$ of asthmatics and refers to the coexistence of hypersensitivity to non-steroidal antiinflammatory drugs, rhinosinusitis/nasal polyps, and asthma [30].

Prostaglandin (PG) $E_{2}$ is the most abundant prostanoid in the lower respiratory tract, produced mainly by epithelial and airway smooth muscle cells. Multiple studies have shown that $\mathrm{PGE}_{2}$ attenuates bronchoconstriction, increases the relaxation of airway smooth muscles and inhibits mast-cell degranulation [26]. PGE 2 activity is expressed not only by direct effect on bronchial smooth muscle relaxation but also as suppression of the inflammatory mediators releasing mainly by eosinophils and mast cells [32]. In AIA diminished level of PGE ${ }_{2}$ was found in nasal polyps [15], peripheral blood cells [27] and bronchial 
fibroblasts [19]. On the contrary, PGD ${ }_{2}$ mast cell autacoid mediator, is a potent agonist for airway smooth muscle contraction.

The $\mathrm{PGE}_{2}$ and $\mathrm{PGD}_{2}$ mediators are chemically unstable and become rapidly converted, via 13,14-dihydro-15-keto$P \mathrm{PE}_{2}$ and $9 a, 11 \beta-\mathrm{PGF}_{2}$, respectively, into tetranor-PGE-M and tetranor-PGD-M. The latter stable metabolites are considered to be the markers of $\mathrm{PGE}_{2}$ and $\mathrm{PGD}_{2}$ systemic production. One of the ultimate dehydration product of $\mathrm{PGD}_{2}$ is 15 -deoxydelta12,14-PGJ, whose level increases under stress such as infection and inflammation.

The inflammatory process in asthma is strongly influenced by leukotriene driven pathways. Leukotriene (LT) $C_{4^{\prime}} \mathrm{LTD}_{4^{\prime}} \mathrm{LTE}_{4}$ are often called cysteinyl leukotrienes (CysLTs). Trans-LTC L $_{4}$ is an isomer of $\mathrm{LTC}_{4}$ [12]. The increased CysLTs level, which may be the result of chronic eosinophlic inflammation of upper and lower respiratory tract, is considered a hallmark of AIA. $\mathrm{LTE}_{4}$, the end-metabolite of CysLTs, is a reliable marker of leukotriene generation in the lung.

Eicosanoids are present in very low concentrations in body fluids and exhaled air. Exhaled breath condensate (EBC) is a non-invasive technique for monitoring airway inflammation in inflammatory diseases such as asthma. Mediators measured in EBC are believed to derive from epithelial lining fluid of the small airways and alveoli [10].

The aim of our study was to evaluate the changes in concentration of wide eicosanoid spectrum in EBC during asthmatic response following histamine inhalation. We used this non-specific agent to assess its influence on inflammation in the airways. The study was carried out in patients with AIA and aspirin-tolerant asthma (ATA). We wondered if clinically manifested bronchoconstriction was accompanied by any change in eicosanoids profile in EBC in asthmatics. To our knowledge, we are the first to perform such investigations.

\section{Material and methods \\ Study subjects}

The study population consisted of 17 asthmatics: 10 AIA subjects and 7 ATA patients. The diagnosis of asthma was established according to Global Initiative for Asthma 2011 update [3]. Hypersensitivity to aspirin was confirmed by an oral aspirin provocation test within 12 months before the study. All ATA patients occasionally used aspirin without any adverse reactions.

Study participants had stable asthma without any exacerbation or respiratory tract infection during 6 weeks preceding the study. Their baseline FEV (forced expiratory volume in 1 second) was $>70 \%$ of predicted value on the day of the study. The subjects were instructed to withdraw medications that decrease bronchial responsiveness before the histamine challenge. Inhaled corticosteroids were allowed at the dose $\leq 1500 \mu \mathrm{g}$ of fluticasone equivalent per day. None of the patients was treated with systemic corticosteroids. The characteristics of the patients are presented in (Table 1).
All the subjects gave informed consent. The study was approved by the University Ethics Committee (KBET/44/B/2009).

\section{Study design}

The single-blind bronchial challenge with histamine [20] began with inhalation of 5 breaths of placebo (saline). Histamine was inhaled every 10 minutes in increasing doses to the cumulative dose $2530 \mu \mathrm{g}$. FEV was measured before, 3 minutes after placebo inhalation and every 3 minutes after each dose of histamine. The challenge procedure was interrupted if $\mathrm{FEV}_{1}$ decreased by more than $20 \%$ of the postsaline baseline value, or if the cumulative dose of histamine was reached. The cumulative dose of histamine causing bronchoconstriction (expressed as $20 \%$ fall in $\mathrm{FEV}_{1}$ ) was recorded as provocative dose (PD20). FEV was assessed after the last dose of histamine every 30 min., until $\mathrm{FEV}_{1}$ reached $>80 \%$ postsaline baseline value.

In all study participants the histamine challenge was positive and $\mathrm{EBC}$ was collected within $30 \mathrm{~min}$. before the challenge and during $30 \mathrm{~min}$. after the time of appearance of bronchoconstriction which was restored spontaneously or was reversed by the inhalation of short-acting $\beta_{2}$-agonist.

\section{Lung function}

Pulmonary function tests were performed using a flowintegrating computerized pneumotachograph (Pneumoscreen, E. Jaeger, Germany).

\section{Exhaled breath condensate}

EBC was collected according to the American Thoracic Society/ European Respiratory Society guidelines [10] using EcoScreen instrument (GmbH Hoechberg, Germany). During 20 minutes of tidal breathing, 2-3 ml of clear fluid was collected and immediately deep frozen.

\section{Biochemical assays}

Concentration of eicosanoids in exhaled breath condensate was measured using gas chromatography/mass spectrometry (GC-MS) and high-performance liquid chromatography/ tandem mass spectrometry (HPLC-MS/MS) according to the methodology described elsewhere $[24,25]$. The results were reported in picograms per milliliter of EBC $(\mathrm{pg} / \mathrm{ml})$, or recalculated as picograms per microgram of palmitic acid content $(\mathrm{pg} / \mathrm{\mu g}$ of PA) due to variable dilution by condensed water.

\section{Statistical analysis}

Descriptive statistics were expressed as mean, standard deviation, median, lower and higher quartile $(25 \%$ and $75 \%$ percentile). General linear model including repeated measures analysis of variance was used for multiple comparisons. Logarithmic transformation was used when needed as variance stabilizing transformation. Correlation between variables was estimated using the Spearman rank order correlations. 
Kaszuba et al. Hypersensitivity 2013,

http://www.hoajonline.com/journals/pdf/2052-594X-1-4.pdf

Table 1. Clinical characteristics of the patients in the subgroups.

\begin{tabular}{|c|c|c|c|c|}
\hline & AIA+ATA $(n=17)$ & $\operatorname{AIA}(\mathbf{n}=7)$ & $\operatorname{ATA}(n=10)$ & p-value AIA vs. ATA \\
\hline \multirow[t]{2}{*}{ Age (yr.) } & $45.9 \pm 11.7$ & $45.3 \pm 11.9$ & $46.3 \pm 12.21$ & 0.87 \\
\hline & $45(35-56)$ & $46(34-57)$ & $44(39-56)$ & \\
\hline Female / Male, no. & $10 / 7$ & $2 / 5$ & $8 / 2$ & 0.051 \\
\hline \multirow[t]{2}{*}{ Duration of astma (yr.) } & $7.82 \pm 6.89$ & $8.7 \pm 5.15$ & $7.2 \pm 8.11$ & 0.28 \\
\hline & $6(3-10)$ & $6(5-14)$ & $3.5(1-10)$ & \\
\hline Inhaled steroids (yes/no), no. & $11 / 6$ & $1 / 6$ & $5 / 5$ & 0.31 \\
\hline Inhaled steroids ( $\mathrm{g}$ of fluticasone & $426.5 \pm 490.3$ & $678.6 \pm 590.1$ & $250 \pm 333.33$ & 0.08 \\
\hline equivalent/d) & $250(0-500)$ & $500(250-1500)$ & $125(0-500)$ & \\
\hline \multirow[t]{2}{*}{ FEV1 baseline (\% predicted) } & $89.64 \pm 10.58$ & $86.79 \pm 10.06$ & $91.64 \pm 11$ & 0.37 \\
\hline & $89.56(82.77-94.44)$ & $85.26(80.53-93.24)$ & $94.25(82.77-97.2)$ & \\
\hline \multirow[t]{2}{*}{ Total IgE (IU/mL) } & $100.9 \pm 149.4$ & $69.44 \pm 30.35$ & $122.86 \pm 194.35$ & 0.46 \\
\hline & $52.4(23-92)$ & $76(46.1-92)$ & $40.5(20-94)$ & \\
\hline Skin prick test (positive/negative), no. & $11 / 6$ & $4 / 3$ & $7 / 3$ & 0.97 \\
\hline \multirow[t]{2}{*}{ Blood eosinophil count (mm-3) } & $315.9 \pm 224.9$ & $477.57 \pm 261.08$ & $202.8 \pm 99.58$ & $0.03^{*}$ \\
\hline & $284(200-339)$ & $484(284-792)$ & $223.5(121-285)$ & \\
\hline \multirow[t]{2}{*}{ NHLBI asthma grade } & $1.29 \pm 0.47$ & $1.4 \pm 0.53$ & $1.2 \pm 0.42$ & 0.35 \\
\hline & $1(1-2)$ & $1(1-2)$ & $1(1-1)$ & \\
\hline \multirow[t]{2}{*}{ ACT } & $22.71 \pm 3.35$ & $20.7 \pm 4.46$ & $24.1 \pm 1.2$ & 0.13 \\
\hline & $24(22-25)$ & $22(16-25)$ & $24.5(24-25)$ & \\
\hline \multirow[t]{2}{*}{ PD20 (mg of histamine) } & $926.5 \pm 709.2$ & $414.29 \pm 320.38$ & $1285 \pm 692.07$ & $0.008^{*}$ \\
\hline & $960(350-1100)$ & $350(155-505)$ & $1100(960-1850)$ & \\
\hline
\end{tabular}

${ }^{*}$ statistical significance values are expressed as mean \pm SD and median with upper and lower quartile (25\% and $75 \%$ percentiles) ACT: Asthma Control Test; AIA: aspirin-induced asthma, ATA: aspirin-tolerant asthma, FEV1: forced expiratory volume in 1 second, IgE: immunoglobulin E, IU: international unit, N.S: not significant, NHLBI: The National Heart, Lung, and Blood Institute; no., number; PD20: provocative dose; y, year.

P-value $<0.05$ was assumed statistically significant.

\section{Results}

\section{Clinical reactions}

During histamine challenge none of the study subjects developed any symptoms after placebo inhalation. All participants responded to histamine. The only statistically significant differences in clinical characteristics between the subjects with AIA and with ATA were higher blood eosinophil count $(p=0.03)$, and lower PD20 $(p=0.008)$ in the AIA group (Table 1).

Exhaled breath condensate concentration of eicosanoids In the studied asthmatics, LTC $_{4}$ and its isomer trans-LTC proved to be the only measured eicosanoids for which EBC concentrations changed following the histamine challenge (Table 2). After the administration of inhaled histamine, their EBC concentration decreased significantly.

At baseline, the AIA group was characterized by higher

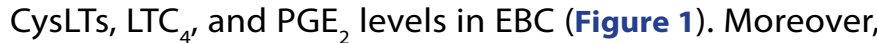
15-deoxy-delta12,14-PGJ ${ }_{2}$ row baseline concentration in
EBC was increased in the AIA group (Table 3). Also, the assessment of changes in eicosanoids $E B C$ concentration after administering histamine enabled us to observe differences between the AIA and ATA groups (Table 3). Following the histamine challenge, the levels of $\mathrm{LTC}_{4}$ (Figure 2), trans-LTC (Figure 3) and tetranor-PGE-M (Figure 4) decreased in the AIA group. None of the measured eicosanoids changed its level after histamine in ATA group.

\section{Correlation between clinical variables and EBC eicosanoids}

Correlation analysis in subgroups was not performed due to the small number of subjects in the groups.

In all studied asthmatics a significant positive correlation between peripheral blood eosinophils and baseline PGE $(r=0.69)$ and $\mathrm{LTC}_{4}(r=0.61)$ concentration in EBC was observed. Baseline FEV1 was negatively correlated with post-challenge $\mathrm{LTE}_{4}(r=-0.63)$ and CysLTs $(r=-0.67)$ EBC concentration. Prechallenge $\mathrm{PGE}_{2}$ correlated significantly with post-challenge one $(r=0.60)$. No significant correlation was found between baseline and post-challenge EBC concentration within other 
Kaszuba et al. Hypersensitivity 2013,

Table 2. Eicosanoids in asthmatic patients at baseline and following histamine challenge.

\begin{tabular}{|c|c|c|c|}
\hline & baseline & challenge & p-value \\
\hline $\mathrm{LTB}_{4}$ & 48.40 & 35.57 & 0.22 \\
\hline$[\mathrm{pg} / \mathrm{mL}]$ & $(33.28-55.28)$ & $(24.37-52.09)$ & \\
\hline $\mathrm{LTB}_{4}$ & 74.64 & 70.42 & 0.71 \\
\hline$[\mathrm{pg} / \mu \mathrm{g}$ of $\mathrm{PA}]$ & $(62.88-94.13)$ & $(42.01-119.02)$ & \\
\hline CysLTs & 5.44 & 4.53 & 0.21 \\
\hline$[\mathrm{pg} / \mathrm{mL}]$ & $(4.52-7.68)$ & $(3.79-6.42)$ & \\
\hline CysLTs & 9.55 & 9.33 & 0.33 \\
\hline$[\mathrm{pg} / \mu \mathrm{g}$ of $\mathrm{PA}]$ & $(8.32-13.72)$ & $(6.99-11.45)$ & \\
\hline $\mathrm{LTC}_{4}$ & 1.71 & 1.14 & $0.04^{*}$ \\
\hline$[\mathrm{pg} / \mathrm{mL}]$ & $(1.21-2.14)$ & $(0.76-1.53)$ & \\
\hline $\mathrm{LTC}_{4}$ & 2.62 & 2.05 & 0.10 \\
\hline$[\mathrm{pg} / \mu \mathrm{g}$ of $\mathrm{PA}]$ & $(2.13-3.44)$ & $(1.19-3.42)$ & \\
\hline trans-LTC 4 & 1.24 & 0.62 & $0.02^{*}$ \\
\hline$[\mathrm{pg} / \mathrm{mL}]$ & $(0.74-2.49)$ & $(0.53-1.11)$ & \\
\hline trans- $\mathrm{LTC}_{4}$ & 2.23 & 1.62 & $0.03^{*}$ \\
\hline$[\mathrm{pg} / \mu \mathrm{g}$ of $\mathrm{PA}]$ & $(1.47-3.71)$ & $(0.75-2.35)$ & \\
\hline $\mathrm{LTD}_{4}$ & 1.09 & 0.93 & 0.64 \\
\hline$[\mathrm{pg} / \mathrm{mL}]$ & $(0.77-1.23)$ & $(0.72-1.23)$ & \\
\hline $\mathrm{LTD}_{4}$ & 1.80 & 1.45 & 0.71 \\
\hline$[\mathrm{pg} / \mu \mathrm{g}$ of $\mathrm{PA}]$ & $(1.12-2.25)$ & $(1.21-2.45)$ & \\
\hline $\mathrm{LTE}_{4}$ & 2.86 & 2.23 & 0.31 \\
\hline$[\mathrm{pg} / \mathrm{mL}]$ & $(1.93-4.18)$ & $(1.62-3.40)$ & \\
\hline $\mathrm{LTE}_{4}$ & 5.22 & 4.33 & 0.76 \\
\hline$[\mathrm{pg} / \mu \mathrm{g}$ of $\mathrm{PA}]$ & $(3.49-6.74)$ & $(3.09-6.18)$ & \\
\hline $\mathrm{PGE}_{2}$ & 1.99 & 2.05 & 0.76 \\
\hline$[\mathrm{pg} / \mathrm{mL}]$ & $(1.44-2.75)$ & $(1.45-2.72)$ & \\
\hline $\mathrm{PGE}_{2}$ & 3.29 & 4.11 & 0.29 \\
\hline$[\mathrm{pg} / \mu \mathrm{g}$ of $\mathrm{PA}]$ & $(2.73-4.65)$ & $(3.32-5.18)$ & \\
\hline 13,14-dihydro-15-keto-PGE2 & 3.49 & 3.83 & 0.67 \\
\hline$[\mathrm{pg} / \mathrm{mL}]$ & $(2.724 .88)$ & $(2.76-4.86)$ & \\
\hline 13,14-dihydro-15-keto-PGE2 & 5.03 & 6.34 & 0.59 \\
\hline$[\mathrm{pg} / \mu \mathrm{g}$ of $\mathrm{PA}]$ & $(4.44-8.03)$ & $(4.77-9.60)$ & \\
\hline tetranor-PGE-M & 21.36 & 15.76 & 0.41 \\
\hline$[\mathrm{pg} / \mathrm{mL}]$ & $(12.20-38.80)$ & $(8.80-23.77)$ & \\
\hline tetranor-PGE-M & 34.50 & 27.64 & 0.71 \\
\hline$[\mathrm{pg} / \mu \mathrm{g}$ of $\mathrm{PA}]$ & $(25.82-49.50)$ & $(16.26-45.38)$ & \\
\hline $\mathrm{PGD}_{2}$ & 1.57 & 1.40 & 0.67 \\
\hline$[\mathrm{pg} / \mathrm{mL}]$ & $(1.29-1.77)$ & $(1.00-1.85)$ & \\
\hline $\mathrm{PGD}_{2}$ & 2.12 & 2.09 & 0.24 \\
\hline$[\mathrm{pg} / \mu \mathrm{g}$ of $\mathrm{PA}]$ & $(1.86-3.03)$ & $(1.94-3.49)$ & \\
\hline
\end{tabular}

Continuation of Table 2.

\begin{tabular}{|c|c|c|c|}
\hline & baseline & challenge & p-value \\
\hline $\begin{array}{l}\text { 13,14-dihydro-15-keto- } \mathrm{PGD}_{2} \\
{[\mathrm{pg} / \mathrm{mL}]}\end{array}$ & $\begin{array}{l}3.48 \\
(2.44-5.03)\end{array}$ & $\begin{array}{l}3.79 \\
(2.44-4.90)\end{array}$ & 0.85 \\
\hline 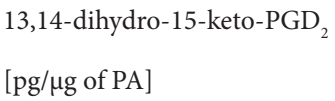 & $\begin{array}{l}6.27 \\
(4.23-7.44)\end{array}$ & $\begin{array}{l}5.88 \\
(4.56-8.71)\end{array}$ & 0.40 \\
\hline $\begin{array}{l}\text { 9a,11b-PGF } \\
{[\mathrm{pg} / \mathrm{mL}](\mathrm{GC}-\mathrm{MS})}\end{array}$ & $\begin{array}{l}0.17 \\
(0.13-0.34)\end{array}$ & $\begin{array}{l}0.11 \\
(0.09-0.26)\end{array}$ & 0.14 \\
\hline 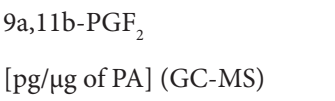 & $\begin{array}{l}0.28 \\
(0.22-0.65)\end{array}$ & $\begin{array}{l}0.22 \\
(0.16-0.53)\end{array}$ & 0.42 \\
\hline $\begin{array}{l}\text { 15-deoxy-delta12,14-PGJ } \\
{[\mathrm{pg} / \mathrm{mL}]}\end{array}$ & $\begin{array}{l}3.58 \\
(1.65-5.06)\end{array}$ & $\begin{array}{l}2.88 \\
(2.34-4.98)\end{array}$ & 0.82 \\
\hline $\begin{array}{l}\text { 15-deoxy-delta12,14-PGJ }{ }_{2} \\
{[\mathrm{pg} / \mu \mathrm{g} \text { of } \mathrm{PA}]}\end{array}$ & $\begin{array}{l}5.54 \\
(3.90-8.60)\end{array}$ & $\begin{array}{l}6.87 \\
(5.10-8.13)\end{array}$ & 0.60 \\
\hline
\end{tabular}

${ }^{*}$ statistical significance values are expressed as median with upper and lower quartile [25\%-75\% percentiles]

AIA: aspirin-induced asthma, ATA: aspirin-tolerant asthma, CysLTs:

cysteinyl leukotrienes, GC-MS: gas chromatography/mass spectrometry, LT:

leukotriene, PA: palmitic acid, PG: prostaglandin.

p-values :

I-AIA vs ATA at baseline.

II-AIA at baseline vs AIA post challenge.

III-ATA at baseline vs ATA post challenge.

measured eicosanoids.

\section{Discussion}

At the beginning of the discussion section, some limitations of the study need to be noted. The main limitation consisted in relatively small patient populations. Another one was the lack of healthy control group. Such a group in the study could have helped us to better assess the production of eicosanoids in the respiratory system of the asthmatics. The study focused mainly on distinguishing between AIA and ATA in this respect.

We have noticed positive significant correlation between peripheral eosinophilia and baseline bronchial production of $\mathrm{LTC}_{4}$ in the studied patients.

The higher level of blood eosinophils was observed in the AIA group, which can probably be connected with decreased eosinophil apoptosis [33]. This observation is in line with previous reports $[11,31]$. The studied AIA subgroup was also characterized by increased baseline concentration of CysLTs and $\mathrm{LTC}_{4}$ in EBC. It is consistent with earlier study using immunoassay [2], although other studies using spectrometric methods did not demonstrate baseline overproduction of CysLTs [22] or LTC 4 [24] in the airways in AIA. However, our observation is in line with researches assessing $\mathrm{LTC}_{4}$ level in induced sputum [18] and can be related to overexpression of LTC synthase (LTCS) in eosinophils [23] and bronchi [21] of asthmatics with aspirin hypersensitivity. Thus, overproduction 
Kaszuba et al. Hypersensitivity 2013,

http://www.hoajonline.com/journals/pdf/2052-594X-1-4.pdf

doi: $10.7243 / 2052-594 X-1-4$

Table 3. Eicosanoids in asthmatic patients at baseline and following histamine challenge in the subgroups.

\begin{tabular}{|c|c|c|c|c|c|c|c|}
\hline & \multicolumn{2}{|c|}{ AIA } & \multicolumn{2}{|c|}{ ATA } & \multicolumn{3}{|c|}{ p-value } \\
\hline & baseline & challenge & baseline & challenge & I & II & III \\
\hline $\begin{array}{l}\mathrm{LTB}_{4} \\
{[\mathrm{pg} / \mathrm{mL}]}\end{array}$ & $\begin{array}{l}50.37 \\
(38.39-97.38)\end{array}$ & $\begin{array}{l}44.16 \\
(24.37-59.99)\end{array}$ & $\begin{array}{l}39.53 \\
(25.11-55.28)\end{array}$ & $\begin{array}{l}30.66 \\
(11.44-47.89)\end{array}$ & 0.39 & 0.74 & 0.90 \\
\hline $\begin{array}{l}\mathrm{LTB}_{4} \\
{[\mathrm{pg} / \mu \mathrm{g} \text { of } \mathrm{PA}]}\end{array}$ & $\begin{array}{l}83.00 \\
(62.88-173.98)\end{array}$ & $\begin{array}{l}78.53 \\
(42.01-119.02)\end{array}$ & $\begin{array}{l}71.11 \\
(54.81-94.13)\end{array}$ & $\begin{array}{l}60.56 \\
(30.56-120.39)\end{array}$ & 0.56 & 0.93 & 1.00 \\
\hline CysLTs & 7.68 & 4.57 & 4.67 & 4.48 & $0.01^{*}$ & 0.07 & 0.99 \\
\hline$[\mathrm{pg} / \mathrm{mL}]$ & $(5.62-14.46)$ & $(4.2-6.42)$ & $(3.52-5.44)$ & $(3.04-6.5)$ & & & \\
\hline CysLTs & 13.72 & 9.85 & 8.46 & 8.6 & $0.02^{*}$ & 0.13 & 0.63 \\
\hline$[\mathrm{pg} / \mu \mathrm{g}$ of $\mathrm{PA}]$ & $(9.55-19.94)$ & $4.97-11.45)$ & $(6.19-10.66)$ & $(7.23-11.63)$ & & & \\
\hline $\mathrm{LTC}_{4}$ & 2.24 & 0.87 & 1.22 & 1.39 & $0.003^{*}$ & $0.001^{*}$ & 1.00 \\
\hline$[\mathrm{pg} / \mathrm{mL}]$ & $(1.90-5.19)$ & $(0.54-1.43)$ & $(0.91-1.44)$ & $(0.76-1.77)$ & & & \\
\hline $\mathrm{LTC}_{4}$ & 3.68 & 1.83 & 2.15 & 2.60 & $0.01^{*}$ & $0.002^{\star}$ & 0.88 \\
\hline$[\mathrm{pg} / \mu \mathrm{g}$ of $\mathrm{PA}]$ & $(3.32-6.62)$ & $(0.83-2.26)$ & $(1.69-2.61)$ & $(1.89-3.71)$ & & & \\
\hline trans- $\mathrm{LTC}_{4}$ & 2.93 & 0.54 & 0.88 & 1.10 & 0.19 & $0.001^{*}$ & 0.83 \\
\hline$[\mathrm{pg} / \mathrm{mL}] \lg$ & $(0.60-4.72)$ & $(0.48-0.62)$ & $(0.74-1.37)$ & $(0.60-1.52)$ & & & \\
\hline 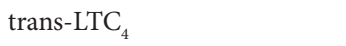 & 4.81 & 0.85 & 2.01 & 2.27 & 0.19 & $0.001^{\star}$ & 0.97 \\
\hline$[\mathrm{pg} / \mu \mathrm{g}$ of PA] & $(1.07-5.88)$ & $(0.62-1.36)$ & $(1.47-2.60)$ & $(1.62-2.94)$ & & & \\
\hline $\mathrm{LTD}_{4}$ & 1.19 & 1.12 & 0.96 & 0.80 & 0.59 & 1.00 & 1.00 \\
\hline$[\mathrm{pg} / \mathrm{mL}]$ & $(0.88-1.65)$ & $(0.83-1.76)$ & $(0.75-1.15)$ & $(0.72-1.07)$ & & & \\
\hline $\mathrm{LTD}_{4}$ & 1.80 & 1.84 & 1.96 & 1.42 & 0.91 & 0.99 & 0.99 \\
\hline$[\mathrm{pg} / \mu \mathrm{g}$ of PA] & $(1.12-2.94)$ & $(1.28-2.72)$ & $(0.92-2.25)$ & $(1.20-2.16)$ & & & \\
\hline $\mathrm{LTE}_{4}$ & 4.18 & 2.80 & 2.31 & 1.79 & 0.22 & 0.58 & 0.97 \\
\hline$[\mathrm{pg} / \mathrm{mL}]$ & $(3.18-7.62)$ & $(2.21-3.40)$ & $(1.49-2.86)$ & $(1.48-4.11)$ & & & \\
\hline $\mathrm{LTE}_{4}$ & 6.74 & 6.08 & 4.08 & 4.32 & 0.42 & 0.73 & 1.00 \\
\hline$[\mathrm{pg} / \mu \mathrm{g}$ of $\mathrm{PA}]$ & $(5.22-9.50)$ & $(2.58-6.74)$ & $(3.17-5.79)$ & $(3.09-5.93)$ & & & \\
\hline $\mathrm{PGE}_{2}$ & 3.22 & 2.84 & 1.58 & 1.58 & $0.004^{*}$ & 0.96 & 1.00 \\
\hline$[\mathrm{pg} / \mathrm{mL}]$ & $(2.23-4.13)$ & $(2.21-7.89)$ & $(1.24-1.94)$ & $(1.15-2.00)$ & & & \\
\hline $\mathrm{PGE}_{2}$ & 4.90 & 5.04 & 2.76 & 3.68 & $0.04^{*}$ & 0.80 & 0.90 \\
\hline$[\mathrm{pg} / \mu \mathrm{g}$ of $\mathrm{PA}]$ & $(3.66-7.08)$ & $(4.11-11.56)$ & $(2.29-3.29)$ & $(2.33-4.34)$ & & & \\
\hline 13,14-dihydro-15-keto-PGE ${ }_{2}$ & 3.82 & 3.83 & 2.73 & 3.46 & 0.83 & 0.91 & 1.00 \\
\hline$[\mathrm{pg} / \mathrm{mL}]$ & $(3.49-4.88)$ & $(2.96-4.35)$ & $(2.26-5.65)$ & $(2.50-5.41)$ & & & \\
\hline 13,14-dihydro-15-keto- $\mathrm{PGE}_{2}$ & 6.55 & 6.34 & 4.64 & 6.22 & 1.00 & 1.00 & 0.88 \\
\hline$[\mathrm{pg} / \mu \mathrm{g}$ of PA] & $(4.65-8.03)$ & $(3.81-8.73)$ & $(3.32-15.98)$ & $(4.77-15.88)$ & & & \\
\hline tetranor-PGE-M & 22.88 & 8.80 & 12.36 & 20.79 & 0.41 & $0.02^{*}$ & 0.23 \\
\hline$[\mathrm{pg} / \mathrm{mL}]$ & $(16.87-38.80)$ & $(8.39-11.14)$ & $(7.97-38.89)$ & $(15.76-32.78)$ & & & \\
\hline tetranor-PGE-M & 36.64 & 14.56 & 33.41 & 41.25 & 0.73 & $0.04^{*}$ & 0.10 \\
\hline$[\mathrm{pg} / \mu \mathrm{g}$ of PA] & $(28.50-49.50)$ & $(13.00-19.42)$ & $(10.92-51.85)$ & $(27.64-87.07)$ & & & \\
\hline $\mathrm{PGD}_{2}$ & 1.48 & 1.48 & 1.58 & 1.23 & 0.99 & 0.99 & 0.90 \\
\hline$[\mathrm{pg} / \mathrm{mL}]$ & $(1.29-1.77)$ & $(1.20-2.36)$ & $(1.28-1.86)$ & $(0.84-1.85)$ & & & \\
\hline $\mathrm{PGD}_{2}$ & 2.52 & 2.29 & 2.04 & 1.98 & 00.99 & 0.86 & 1.00 \\
\hline$[\mathrm{pg} / \mu \mathrm{g}$ of $\mathrm{PA}]$ & $(1.89-3.03)$ & $(1.99-5.19)$ & $(1.76-4.80)$ & $(1.64-3.49)$ & & & \\
\hline 13,14-dihydro-15-keto-PGD 2 & 5.03 & 4.81 & 2.91 & 2.92 & 0.24 & 0.93 & 0.99 \\
\hline$[\mathrm{pg} / \mathrm{mL}]$ & $(4.19-5.63)$ & $(3.52-4.92)$ & $(1.95-3.48)$ & $(2.16-4.21)$ & & & \\
\hline 13,14-dihydro-15-keto-PGD 2 & 7.18 & 6.64 & 4.97 & 5.88 & 0.82 & 1.00 & 0.67 \\
\hline
\end{tabular}


Kaszuba et al. Hypersensitivity 2013,

http://www.hoajonline.com/journals/pdf/2052-594X-1-4.pdf

Continuation of Table 3.

\begin{tabular}{|c|c|c|c|c|c|c|c|}
\hline & \multicolumn{2}{|c|}{ AIA } & \multicolumn{2}{|c|}{ ATA } & \multicolumn{3}{|c|}{ p-value } \\
\hline & baseline & challenge & baseline & challenge & I & II & III \\
\hline 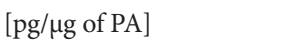 & $(6.27-9.33)$ & $(5.44-9.86)$ & $(4.04-7.44)$ & $(3.90-8.71)$ & & & \\
\hline $\begin{array}{l}\text { 9a,11b-PGF } \\
{[\mathrm{pg} / \mathrm{mL}](\mathrm{GC}-\mathrm{MS})}\end{array}$ & $\begin{array}{l}0.22 \\
(0.14-0.38)\end{array}$ & $\begin{array}{l}0.10 \\
(0.08-0.71)\end{array}$ & $\begin{array}{l}0.16 \\
(0.09-0.28)\end{array}$ & $\begin{array}{l}0.12 \\
(0.09-0.14)\end{array}$ & 0.88 & 0.91 & 0.52 \\
\hline $\begin{array}{l}\text { 9a,11b-PGF } \\
{[\mathrm{pg} / \mu \mathrm{g} \text { of PA] (GC-MS) }}\end{array}$ & $\begin{array}{l}0.30 \\
(0.25-0.65)\end{array}$ & $\begin{array}{l}0.20 \\
(0.14-1.23)\end{array}$ & $\begin{array}{l}0.24 \\
(0.18-0.70)\end{array}$ & $\begin{array}{l}0.23 \\
(0.16-0.39)\end{array}$ & 0.99 & 0.99 & 0.85 \\
\hline $\begin{array}{l}\text { 15-deoxy-delta12,14-PGJ }{ }_{2} \\
{[\mathrm{pg} / \mathrm{mL}]}\end{array}$ & $\begin{array}{l}5.55 \\
(4.24-8.36)\end{array}$ & $\begin{array}{l}3.30 \\
(2.12-4.98)\end{array}$ & $\begin{array}{l}2.55 \\
(1.43-3.58)\end{array}$ & $\begin{array}{l}2.84 \\
(2.34-5.02)\end{array}$ & $0.04^{\star}$ & 0.43 & 0.41 \\
\hline $\begin{array}{l}\text { 15-deoxy-delta12,14-PGJ }{ }_{2} \\
{[\mathrm{pg} / \mu \mathrm{g} \text { of } \mathrm{PA}]}\end{array}$ & $\begin{array}{l}9.13 \\
(5.28-10.66)\end{array}$ & $\begin{array}{l}5.40 \\
(3.66-8.33)\end{array}$ & $\begin{array}{l}4.58 \\
(2.52-5.84)\end{array}$ & $\begin{array}{l}7.11 \\
(5.51-8.13)\end{array}$ & 0.09 & 0.73 & 0.32 \\
\hline
\end{tabular}

* statistical significance values are expressed as median with upper and lower quartile [25\%-75\% percentiles]

AIA: aspirin-induced asthma, ATA: aspirin-tolerant asthma, CysLTs: cysteinyl leukotrienes, GC-MS: gas chromatography/mass spectrometry, LT: leukotriene, PA: palmitic acid, PG: prostaglandin.

p-values :

I - AIA vs ATA at baseline.

II - AIA at baseline vs AIA post challenge.

III - ATA at baseline vs ATA post challenge.

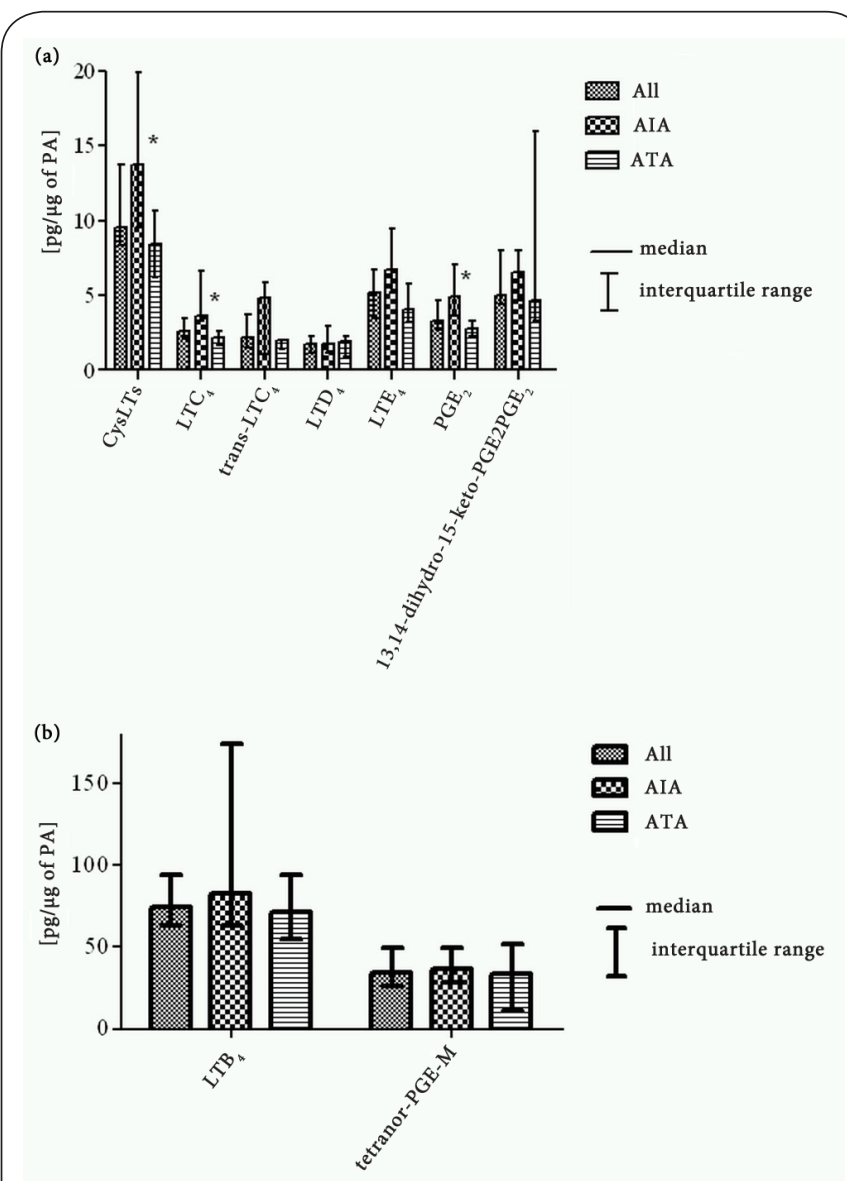

Figure 1. (a, b)Eicosanoids' concentrations in EBC. AIA: aspirin induced asthma, ATA: aspirin tolerant asthma, EBC: exhaled breath condensate, post: after histamine, pre: before histamine; ${ }^{*}, \mathrm{p}<0.05$.
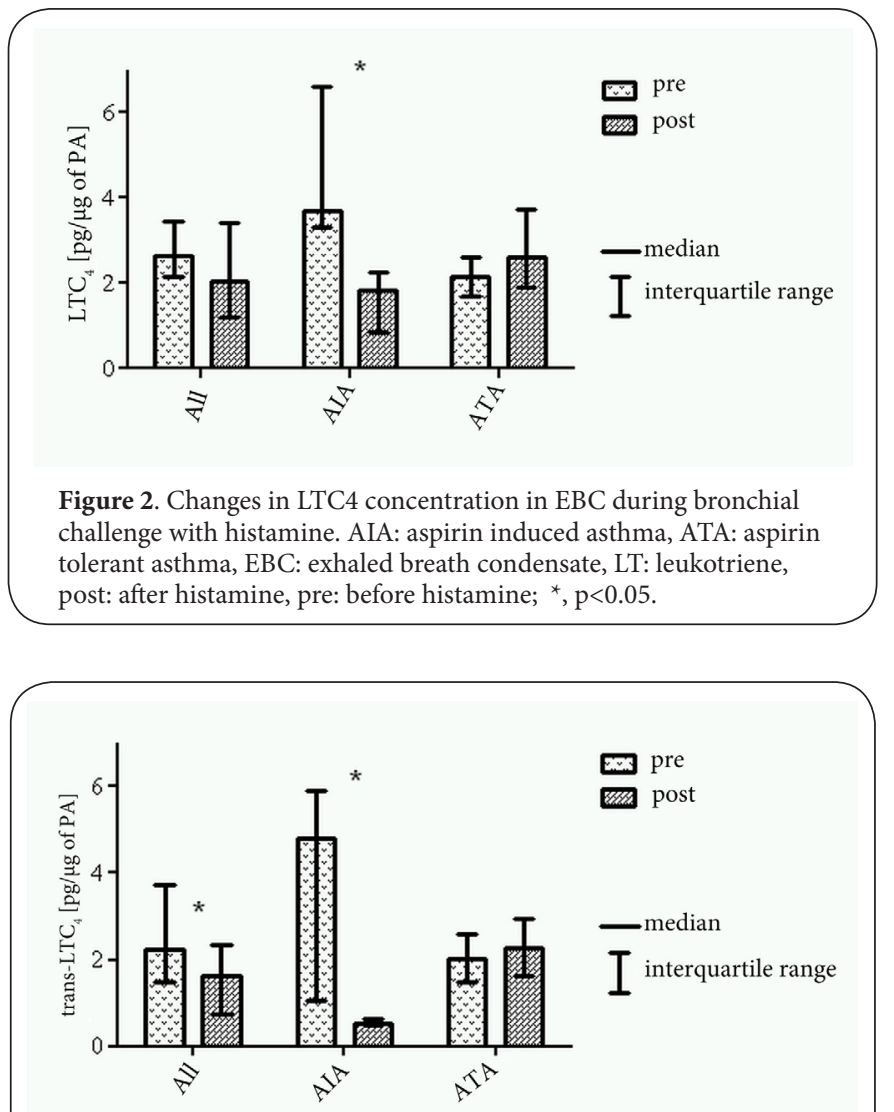

Figure 3. Changes in trans-LTC concentration in EBC during bronchial challenge with histamine. AIA: aspirin induced asthma, ATA: aspirin tolerant asthma, EBC: exhaled breath condensate; transLT: trans-leukotriene, post: after histamine, pre: before histamine; ${ }^{*}$, $\mathrm{p}<0.05$. 


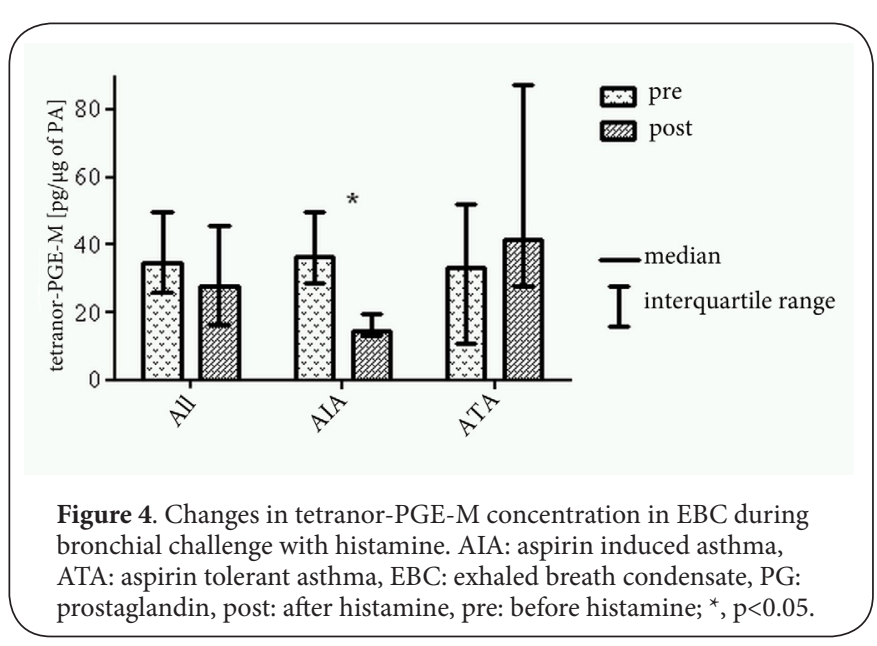

of LTC observed in our study in AIA may be the result of both overexpression of LTCS and overrepresentation of eosinophils. Other important sources of eicosanoids could also be taken into consideration while explaining these observations, such as mast cells and airway epithelial cells, which were not assessed in the study.

The elevated level of $\mathrm{PGE}_{2}$ in $\mathrm{EBC}$ in the AIA group was a surprising finding. Other publications suggest diminished production of $\mathrm{PGE}_{2}$ in peripheral blood cells, upper airways and bronchial fibroblasts [30] in AIA. However the level of $\mathrm{PGE}_{2}$ in BALF after placebo inhalation was higher in patients with AIA [28]. Increased production of PGE, by basophil-enriched leukocytes in AIA patients has also been reported [8].

Bronchoconstriction appeared in both groups, which probably is an effect of direct histamine action on airway smooth muscle via histamine receptors. Interestingly, the level of PD20 was much lower in AIA than in ATA group. It may indicate more severe bronchial inflammation in asthmatics sensitive to aspirin. The elevated levels of Cys LTs, $\mathrm{LTC}_{4^{\prime}} \mathrm{PGE}_{2}$ and 15-deoxy-delta12,14-PGJ in EBC of the AIA patients can be observed in our study as the result of such a condition. This state can be mirrored also in reported more severe clinical course of AIA, although differences between studied subgroups in clinical parameters are not statistically significant.

During histamine challenge, we noticed a decrease in EBC concentration of LTC $_{4}$ and its isomer only in AIA group without significant changes in the levels of the other cysteinyl leukotrienes (assessed separately or totally). In a previous study, we showed that during bronchial challenge with specific agent=aspirin=the concentration of $\mathrm{LTC}_{4}$ decreased also only in AIA group. In that study in both groups, despite increase of $\mathrm{LTE}_{4}$ level, the total CysLTs concentration in EBC remained unchanged [16]. However, in many studies the levels of CysLTs was increased in AIA subjects following aspirin challenge in various biological samples $[6,7,9,14,29]$. Our previous and present observations may be explained by enhanced transformation of $\mathrm{LTC}_{4}$ via $\mathrm{LTD}_{4}$ to stable $\mathrm{LTE}_{4}$.
Secondly, inflammatory mediators, such as $\mathrm{LTE}_{4^{\prime}}$ are released and act mainly across the distance of a few micrometers [5] on adjacent cells and, to a lesser extent, are excreted into the lumen of bronchi, so their measurement in EBC might be biased. The difference between $\mathrm{LTC}_{4}$ and $\mathrm{LTE}_{4}$ in terms of excretion into the bronchial lumen needs further exploration. It is difficult to explain decreased concentration of tetranorPGE-M in EBC after histamine during bronchial challenge. Our observation may suggest diminished synthesis of $\mathrm{PGE}_{2}$ under the influence of histamine, or what is less probable, decreased metabolism of this prostanoid.

\section{Conclusions}

The aim of this study was to assess the indirect effect of locally administrated stimulus on lower airways by measuring wide profile of eicosanoids released to the bronchi and present in EBC during the bronchial histamine challenge. In our study we demonstrated that histamine triggers not only direct but also indirect effects in respiratory tract causing both bronchoconstriction and alterations in the eicosanoid synthesis in the lungs. We demonstrated for the first time changes in exhaled eicosanoids in asthmatics after inhalation of histamine. This indirect action of histamine let us to reveal discrepancies in asthmatic inflammation between subjects with hypersensitivity to aspirin and tolerating aspirin well.

\section{Competing interests}

The authors declare that they have no competing interests.

Authors' contributions

\begin{tabular}{|l|c|c|c|c|c|c|c|}
\hline Authors' contributions & MK & JK & AS & AK & PS & MS & LM \\
\hline Research concept and design & $\checkmark$ & -- & -- & -- & $\checkmark$ & -- & $\checkmark$ \\
\hline Collection and/or assembly of data & $\checkmark$ & -- & $\checkmark$ & $\checkmark$ & -- & $\checkmark$ & -- \\
\hline Data analysis and interpretation & -- & $\checkmark$ & -- & -- & -- & -- & -- \\
\hline Writing the article & $\checkmark$ & $\checkmark$ & -- & -- & -- & -- & -- \\
\hline Critical revision of the article & -- & -- & -- & -- & $\checkmark$ & -- & $\checkmark$ \\
\hline Final approval of article & -- & -- & -- & -- & -- & -- & $\checkmark$ \\
\hline Statistical analysis & $\checkmark$ & -- & -- & -- & -- & -- & - \\
\hline
\end{tabular}

Acknowledgement

Presented study was supported by the grant K/PBW/0000552 from the Polish Ministry of Science and Higher Education. We wish to thank Anna Gielicz M.Sc. for completing the measurement of eicosanoids using mass spectrometry. The authors are grateful also to the subjects for their participation in the study.

\section{Publication history}

Editor: Garry M. Walsh, University of Aberdeen, UK.

EIC: Yoshimichi Okayama, Nihon University

Graduate School of Medical Sciences, Japan

Received: 02-Sep-2013 Revised: 11-Oct-2013

Accepted: 20-Nov-2013 Published:27-Dec-2013 
Kaszuba et al. Hypersensitivity 2013,

\section{References}

1. Amin K. The role of mast cells in allergic inflammation. Respir Med. 2012; 106:9-14. | Article | PubMed

2. Antczak A, Montuschi P, Kharitonov S, Gorski P and Barnes PJ. Increased exhaled cysteinyl-leukotrienes and 8-isoprostane in aspirin-induced asthma. Am J Respir Crit Care Med. 2002; 166:301-6. I Article I PubMed

3. Bateman ED, Boulet LP, Cruz AA, FitzGerald M, Haahtela T, Levy ML, O'Byrne P, Ohta K, Piaggiaro P, Pedersen SE, Soto-Quiro M and Wong GW. Global strategy for asthma management and prevention. Updated 2011 $2011 \mid$ Pdf

4. Beasley R, Roche WR, Roberts JA and Holgate ST. Cellular events in the bronchi in mild asthma and after bronchial provocation. Am Rev Respir Dis. 1989; 139:806-17. | Article | PubMed

5. Bradding $P$ and Brightling $C$. Mast cell infiltration of airway smooth muscle in asthma. Respir Med. 2007; 101:1045; author reply 1046-7. | Article | PubMed

6. Christie PE, Tagari P, Ford-Hutchinson AW, Charlesson S, Chee P, Arm JP and Lee TH. Urinary leukotriene E4 concentrations increase after aspirin challenge in aspirin-sensitive asthmatic subjects. Am Rev Respir Dis. 1991; 143:1025-9. | Article | PubMed

7. Gaber F, Daham K, Higashi A, Higashi N, Gulich A, Delin I, James A, Skedinger M, Gyllfors P, Nord M, Dahlen SE, Kumlin M and Dahlen B. Increased levels of cysteinyl-leukotrienes in saliva, induced sputum, urine and blood from patients with aspirin-intolerant asthma. Thorax. 2008; 63:1076-82. | Article | PubMed

8. Goetzl EJ, Valacer DJ, Payan DG and Wong MY. Abnormal responses to aspirin of leukocyte oxygenation of arachidonic acid in adults with aspirin intolerance. J Allergy Clin Immunol. 1986; 77:693-8. | Article | PubMed

9. Higashi N, Taniguchi M, Mita H, Osame M and Akiyama K. A comparative study of eicosanoid concentrations in sputum and urine in patients with aspirin-intolerant asthma. Clin Exp Allergy. 2002; 32:1484-90. | Article | PubMed

10. Horvath I, Hunt J, Barnes PJ, Alving K, Antczak A, Baraldi E, Becher G, van Beurden WJ, Corradi M, Dekhuijzen R, Dweik RA, Dwyer T, Effros R, Erzurum S, Gaston B, Gessner C, Greening A, Ho LP, Hohlfeld J, Jobsis Q, Laskowski D, Loukides S, Marlin D, Montuschi P, Olin AC, Redington $A E$, Reinhold $P$, van Rensen EL, Rubinstein I, Silkoff P, Toren K, Vass G, Vogelberg $\mathrm{C}$ and Wirtz $\mathrm{H}$. Exhaled breath condensate: methodological recommendations and unresolved questions. Eur Respir J. 2005; 26:52348. | Article | PubMed

11. Jawien J. A new insight into aspirin-induced asthma. Eur J Clin Invest. 2002; 32:134-8. | Article | PubMed

12. Jorg A, Henderson WR, Murphy RC and Klebanoff SJ. Leukotriene generation by eosinophils. J Exp Med. 1982; 155:390-402. | Article | PubMed Abstract I PubMed Full Text

13. Kirby JG, Hargreave FE, Gleich GJ and O'Byrne PM. Bronchoalveolar cell profiles of asthmatic and nonasthmatic subjects. Am Rev Respir Dis. 1987; 136:379-83. | Article | PubMed

14. Kowalski ML. Aspirin-sensitive rhinosinusitis and asthma. Clin Allergy Immunol. 2007; 19:147-75. | PubMed

15. Kowalski ML, Pawliczak R, Wozniak J, Siuda K, Poniatowska M, Iwaszkiewicz J, Kornatowski T and Kaliner MA. Differential metabolism of arachidonic acid in nasal polyp epithelial cells cultured from aspirinsensitive and aspirin-tolerant patients. Am J Respir Crit Care Med. 2000; 161:391-8. | Article | PubMed

16. Mastalerz L, Sanak M, Kumik J, Gawlewicz-Mroczka A, Celejewska-Wójcik $\mathrm{N}$, Cmiel A and Szczeklik A. Exhaled Eicosanoids following Bronchial Aspirin Challenge in Asthma Patients with and without Aspirin Hypersensitivity: The Pilot Study. J. Allergy. 2012; 2012:696792. | Article

17. $\overline{O^{\prime} B y r n e}$ PM. Conclusion: Airway hyperresponsiveness in asthma: its measurement and clinical significance. Chest. 2010; 138:44S-45S. I Article | PubMed

18. Obase Y, Shimoda T, Tomari SY, Mitsuta K, Kawano T, Matsuse H and Kohno $S$. Effects of pranlukast on chemical mediators in induced sputum on provocation tests in atopic and aspirin-intolerant asthmatic patients. Chest. 2002; 121:143-50. | Article | PubMed

19. Pierzchalska M, Szabo Z, Sanak M, Soja J and Szczeklik A. Deficient prostaglandin E2 production by bronchial fibroblasts of asthmatic patients, with special reference to aspirin-induced asthma. J Allergy Clin Immunol. 2003; 111:1041-8. | Article | PubMed
20. Ryan G, Dolovich MB, Roberts RS, Frith PA, Juniper EF, Hargreave FE and Newhouse MT. Standardization of inhalation provocation tests: two techniques of aerosol generation and inhalation compared. Am Rev Respir Dis. 1981; 123:195-9. | PubMed

21. Sampson AP, Cowburn AS, Sladek K, Adamek L, Nizankowska E, Szczeklik A, Lam BK, Penrose JF, Austen KF and Holgate ST. Profound overexpression of leukotriene $\mathrm{C} 4$ synthase in bronchial biopsies from aspirin-intolerant asthmatic patients. Int Arch Allergy Immunol. 1997; 113:355-7. | Article | PubMed

22. Sanak M, Kielbasa B, Bochenek $G$ and Szczeklik A. Exhaled eicosanoids following oral aspirin challenge in asthmatic patients. Clin Exp Allergy. 2004; 34:1899-904. | Article | PubMed

23. Sanak M, Pierzchalska M, Bazan-Socha S and Szczeklik A. Enhanced expression of the leukotriene $C(4)$ synthase due to overactive transcription of an allelic variant associated with aspirin-intolerant asthma. Am J Respir Cell Mol Biol. 2000; 23:290-6. | Article | PubMed

24. Sanak M, Gielicz A, Bochenek G, Kaszuba M, Nizankowska-Mogilnicka $E$ and Szczeklik A. Targeted eicosanoid lipidomics of exhaled breath condensate provide a distinct pattern in the aspirin-intolerant asthma phenotype. J Allergy Clin Immunol. 2011; 127:1141-7. | Article | PubMed

25. Sanak M, Gielicz A, Nagraba K, Kaszuba M, Kumik J and Szczeklik A. Targeted eicosanoids lipidomics of exhaled breath condensate in healthy subjects. J Chromatogr B Analyt Technol Biomed Life Sci. 2010; 878:1796-800. | Article | PubMed

26. Sastre $B$ and del Pozo V. Role of PGE2 in asthma and nonasthmatic eosinophilic bronchitis. Mediators Inflamm. 2012; 2012:645383. | Article | PubMed Abstract | PubMed Full Text

27. Schafer D, Schmid M, Gode UC and Baenkler HW. Dynamics of eicosanoids in peripheral blood cells during bronchial provocation in aspirin-intolerant asthmatics. Eur Respir J. 1999; 13:638-46. | Article | PubMed

28. Sladek K, Dworski R, Soja J, Sheller JR, Nizankowska E, Oates JA and Szczeklik A. Eicosanoids in bronchoalveolar lavage fluid of aspirinintolerant patients with asthma after aspirin challenge. Am J Respir Crit Care Med. 1994; 149:940-6. | Article | PubMed

29. Szczeklik A, Sladek K, Dworski R, Nizankowska E, Soja J, Sheller J and Oates J. Bronchial aspirin challenge causes specific eicosanoid response in aspirin-sensitive asthmatics. Am J Respir Crit Care Med. 1996; 154:1608-14. | Article | PubMed

30. Szczeklik A., Niżankowska-Mogilnicka E and Sanak M: Hypersensitivity to aspirin and nonsteroidal anti-inflammatory drugs. In: Adkinson NF, Busse WW, Bochner BS, Holgate ST, Simons FER, Lemanske RF (Eds.), Middelton's allergy: principles and practice 2009; 1227-43.

31. Szczeklik A and Stevenson DD. Aspirin-induced asthma: advances in pathogenesis, diagnosis, and management. J Allergy Clin Immunol. 2003; 111:913-21. | Article | PubMed

32. Tanaka $\mathrm{H}$, Kanako $\mathrm{S}$ and Abe S. Prostaglandin E2 receptor selective agonists E-prostanoid 2 and E-prostanoid 4 may have therapeutic effects on ovalbumin-induced bronchoconstriction. Chest. 2005; 128:3717-23. | Article | PubMed

33. Ziroli NE, Na H, Chow JM, Stankiewicz JA, Samter M and Young MR. Aspirin-sensitive versus non-aspirin-sensitive nasal polyp patients: analysis of leukotrienes/Fas and Fas-ligand expression. Otolaryngol Head Neck Surg. 2002; 126:141-6. | Article | PubMed

\section{Citation:}

Kaszuba M, Kaszuba J, Sawina A, Kleciek A, Stręk P, Sanak $M$ and Mastalerz L. Eicosanoids in exhaled air after non-specific challenge in patients with asthma. Hypersensitivity. 2013; 1:4. http://dx.doi.org/10.7243/2052-594X-1-4 